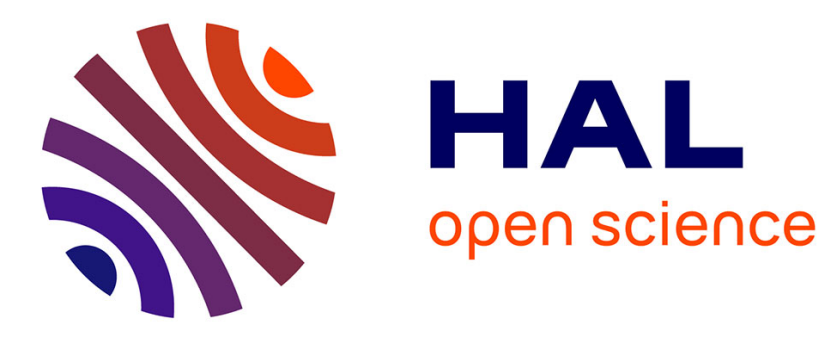

\title{
Oxygen adsorption on the Al9Co2(001) surface: first-principles and STM study
}

S. Alarcon Villaseca, L. Serkovic Loli, J. Ledieu, V. Fournée, P. Gille, J.-M. Dubois, Emilie Gaudry

\section{- To cite this version:}

S. Alarcon Villaseca, L. Serkovic Loli, J. Ledieu, V. Fournée, P. Gille, et al.. Oxygen adsorption on the Al9Co2(001) surface: first-principles and STM study. Journal of Physics: Condensed Matter, 2013, 25, pp.355003. 10.1088/0953-8984/25/35/355003 . hal-00977985

\section{HAL Id: hal-00977985 \\ https://hal.science/hal-00977985}

Submitted on 20 Oct 2014

HAL is a multi-disciplinary open access archive for the deposit and dissemination of scientific research documents, whether they are published or not. The documents may come from teaching and research institutions in France or abroad, or from public or private research centers.
L'archive ouverte pluridisciplinaire HAL, est destinée au dépôt et à la diffusion de documents scientifiques de niveau recherche, publiés ou non, émanant des établissements d'enseignement et de recherche français ou étrangers, des laboratoires publics ou privés. 


\title{
Oxygen adsorption on the $\mathrm{Al}_{9} \mathrm{Co}_{2}(001)$ surface: first-principles and STM study
}

\author{
S. Alarcón Villaseca ${ }^{1}$, L.N. Serkovic Loli ${ }^{1}$, J. Ledieu ${ }^{1}$, V. \\ Fournée $^{1}$, P. Gille ${ }^{2}$, J.-M. Dubois ${ }^{1}$ and É. Gaudry ${ }^{1}$ \\ ${ }^{1}$ Institut Jean Lamour, UMR 7198 (CNRS Université de Lorraine), Parc de Saurupt, \\ 54011 Nancy Cedex, France. ${ }^{2}$ Department of Earth and Environmental Science, \\ Crystallography Section, LMU, Theresienstr. 41, D-80333 München, Germany. \\ E-mail: Emilie.Gaudry@ijl.nancy-universite.fr
}

\begin{abstract}
Atomic oxygen adsorption on the pure aluminium terminated $\mathrm{Al}_{9} \mathrm{Co}_{2}(001)$ surface is studied by first principle calculations coupled with STM measurements. Relative adsorption energy of oxygen atoms have been calculated on different surface sites along with the associated STM images. The local electronic structure of the most favourable adsorption site is described. The preferential adsorption site is identified as a "bridge" type site between the cluster entities exposed at the (001) surface termination. The Al-O bonding between the adsorbate and the substrate presents a covalent character, with $s-p$ hybridization occuring between the states of the adsorbed oxygen atom and the aluminium atoms of the surface. The simulated STM image of the preferential adsorption site is in agreement with experimental observations. This work shows that oxygen adsorption generates important atomic relaxations of the topmost surface layer and that sub-surface cobalt atoms strongly influence the values of the adsorption energies. The calculated $\mathrm{Al}-\mathrm{O}$ distances are in agreement with those reported in $\mathrm{Al}_{2} \mathrm{O}$ and $\mathrm{Al}_{2} \mathrm{O}_{3}$ oxides and for oxygen adsorption on $\mathrm{Al}(111)$.
\end{abstract}

PACS numbers: 68.43.Mn, 68.35.B-, 71.20.Gj, 71.15.Mb, 61.44.Br, 68.43.Bc

Keywords: surface science, density functional theory, complex metallic alloy, oxygen adsorption, scanning tunnelling microscopy 


\section{Introduction}

Aluminium-based oxide barriers are widely used in the manufacture of stable coatings which are typically resistant to high temperature and corrosive environments [1]. These oxide barriers are also at the origin of the loss of performance of heterogeneous catalysts working during long term exposition under real industrial conditions [2]. From these two examples, the study of the interaction of oxygen with metallic surfaces appears vital in the comprehension of a broad range of chemical processes of technological relevance.

The low resistance of metallic surfaces towards oxidation is related to the strong negative values of the enthalpies of formation of the oxides, e.g. for the oxide $\mathrm{Al}_{2} \mathrm{O}_{3}$ the enthalpy of formation is $\Delta H_{f}=-1675 \mathrm{~kJ} / \mathrm{mol}$, which means that oxide phases are energetically more favourable $[2,3]$. The oxidation of a metallic surface is a complex phenomenon that involves different steps. In a general way, it has been established that the first step of surface oxidation is the dissociation of the adsorbed $\mathrm{O}_{2}$ molecules. Experimentally, measurements of the probability of dissociation show that the $\mathrm{O}_{2}$ molecule dissociates on metallic surfaces $[4,5]$. Here, the reaction yield of the dissociative reaction increases with the kinetic energy up to saturation at $\simeq 0.6 \mathrm{eV}$ $[4,5]$. This process is followed by the adsorption of the dissociated oxygen atoms on the substrate, which form strong bonds with the atoms at the surface [4]. In a high number of metallic surfaces, the preferential adsorption sites typically correspond to atomic surface environments of symmetry 3 or 4 , referred to hollow sites. The sticking probability $\left(P_{S}\right)$ of an oxygen atom on the surface of a metal is high, e.g. at $300 \mathrm{~K} P_{S}$ is of the order of 1 for $\mathrm{Ni}$ and $\mathrm{Co}$ [3]. Surface dissociation and penetration of oxygen in the sub-surface of the solid may also occur and they can generate reconstructions of the surface atomic structure as well as the formation of an oxide phase in the topmost layers [4]. Similar to the pure aluminium surfaces, oxygen adsorption on the surfaces of Al-TM (TM = transition metal) alloys generally leads to the formation of $\mathrm{Al}_{2} \mathrm{O}_{3}$ oxide layers in the surface. The latter is related to the larger stability of the $\mathrm{Al}_{2} \mathrm{O}_{3}$ oxide compared to the transition metal oxides [1]. Oxygen adsorption on the (100) surface of the equi-atomic AlCo intermetallic compound allows to illustrate this point. In the study of $\mathrm{V}$. Rose et al. [6], it is demonstrated that the formation at $300 \mathrm{~K}$ of an $\mathrm{Al}_{2} \mathrm{O}_{3}$ layer is thermodynamically more favourable $\left(\Delta H_{f}=-1675 \mathrm{~kJ} / \mathrm{mol}\right)$ than the formation of the $\mathrm{CoO}\left(\Delta H_{f}=-238 \mathrm{~kJ} / \mathrm{mol}\right)$ and $\mathrm{Co}_{3} \mathrm{O}_{4}\left(\Delta H_{f}=-891 \mathrm{~kJ} / \mathrm{mol}\right)$ oxides. This study also shows that the $\mathrm{AlCo}(100)$ surface is completely covered by amorphous $\mathrm{Al}_{2} \mathrm{O}_{3}$ [6]. The situation is similar in the low-index surfaces of the $\mathrm{AlNi}, \mathrm{AlNi}_{3}, \mathrm{GaCo}$ and $\mathrm{AlFe}$ alloys: the formation of an $\mathrm{Al}_{2} \mathrm{O}_{3}\left(\mathrm{Ga}_{2} \mathrm{O}_{3}\right)$ layer is favoured $[7,8,9]$.

One of the most widely used methods to determine the adsorption sites of oxygen atoms in metallic surfaces is scanning tunnelling microscopy (STM). Nevertheless, the interpretation of the experimentally recorded STM images is delicate. Even in the simple case of $\mathrm{O}_{2}$ adsorption on a $\mathrm{Al}(111)$ surface, fundamental questions remain open, particularly regarding the dissociation process of the $\mathrm{O}_{2}$ molecule $[10,11]$. The work of H. Brune et al. $[12,13]$ show that atomic oxygen on $\mathrm{Al}(111)$ appear at dark contrast in 
STM images recorded at room temperature. It is attributed to the presence of atomic oxygen in fcc sites. Moreover, it is claimed that the distance of separation between both atoms of a dissociated $\mathrm{O}_{2}$ molecule at room temperature on the $\mathrm{Al}(111)$ surface is of the order of $80 \AA$. The authors called this effect "surface migration of hot adatoms" [12, 13]. Later studies demonstrated that the oxygen atoms from a dissociated $\mathrm{O}_{2}$ molecule were diffusing distances of 2 or 3 times the surface lattice parameter, with a distance of separation of about 10-15 $\AA[4,10,11,14,15]$. In the work of M. Schmid et al. [10], the dark contrast observed in STM images was attributed to groups of atoms on the surface, i.e. dimers, trimers, etc. Differences with previous works were explained by the interaction between the STM tip and the sample. At lower temperatures $(80 \mathrm{~K})$, the STM images show the presence of dark contrast forming pairs of black dots [10]. Some of these dark dots are sometimes lengthened according to the low index directions of the surface and they are interpreted as pairs of oxygen atoms, placed each one in adjacent fcc hollow sites. Nevertheless, the STM images also show pairs of black dots for which neither the direction nor the distance of separation are compatible with two oxygen atoms occupying fcc sites [10]. So far, several models have been elaborated to explain these observations. One model involves oxygen atoms negatively charged that describe ballistic trajectories [4]. A second model has been proposed to attribute a different role to the two dissociated oxygen atoms: one of the atoms of the $\mathrm{O}_{2}$ molecule forms a bond with the surface and the other atom is ejected ("canon ball motion") [10]. These examples show that even in simple cases, like the dissociation of the $\mathrm{O}_{2}$ molecule on the $\mathrm{Al}(111)$ surface, the interpretation of STM images recorded during the oxygen adsorption is not straightforward, partly because STM images are linked to the electronic structure of the surface and do not image directly the atomic positions of the surface atoms. In the case of alloys, the understanding of the oxidation process is expected to show similar trends: generally the adsorbed oxygen atoms appear as depressions in the STM images (dark contrast) [6, 8, 9, 16, 17, 18]. For example, in the oxidation of the $\operatorname{PdAg}(111)$ surface, the clear contrast zones observed in the STM images of the clean surface correspond to palladium atoms, which become dark in response to the adsorption of atomic oxygen [16]. This can be interpreted as a selective adsorption of oxygen atoms on $\mathrm{Pd}$ sites isolated by $\mathrm{Ag}$ atoms [16].

The study of the oxidation of metallic surfaces constitutes a challenge in surface science, since the interaction between a surface and an adsorbate can induce important structural modifications under ultra high vacuum conditions [2]. To understand the mechanisms of the different steps towards oxide surface formation, an analysis at atomistic scale is essential. An approach combining experimental STM analysis and firstprinciples STM images, simulated from model structures within the Tersoff-Hamann approximation $[19,20]$, represents a powerful tool to study the first steps of the oxidation. In this article, we focus on the adsorption of atomic oxygen on the (001) surface of the intermetallic compound $\mathrm{Al}_{9} \mathrm{Co}_{2}$. The $\mathrm{Al}_{9} \mathrm{Co}_{2}$ crystal is an intermetallic with an intermediate structural complexity between the B2-AlCo and the decagonal Al-Ni-Co quasicrystal. Al-based intermetallic compounds have been attracting great 
research interest regarding applications, since they combine good mechanical properties and low density $[21,22,1,23,24]$. As a complex metallic alloy, the electronic structure of $\mathrm{Al}_{9} \mathrm{Co}_{2}$ includes a pseudogap near the Fermi energy [25], which suggests that this material could present a superior corrosion resistance, like quasicrystals, compared to simpler alloys [26, 27, 28, 29]. The (001) surface is bulk truncated, with the selection of a pure aluminium layer as terminating plane. Although the topmost layer is a pure aluminium termination, the sub-layer contains cobalt atoms that can play an important role in the adsorption properties and reactivity of the surface.

The paper is organized as follows. Calculations and experimental details are gathered in section 2. The identification of the most favoured adsorption sites, the analysis of the bonding between atomic oxygen and the surface and the confrontation of the theoretical results with experimental observations are presented and discussed in section 3 before we conclude.

\section{Calculation and experimental details}

\subsection{Calculation details}

Calculations are performed using the Vienna ab initio Simulation Package (VASP) based on the Density Functional Theory (DFT) [30, 31]. The interaction between the valence electrons and the ionic core is described using the projector-augmented wave (PAW) method [32, 33]. The electronic exchange and correlation contribution is described using the generalized gradient approximation GGA-PBE [34, 35]. Geometries are optimized by the calculation of the Hellmann-Feynman forces acting on atoms and their minimization via a conjugate gradient algorithm. The parameters linked to the numerical implementation of the DFT, i.e. the plane-wave cutoff $E_{c u t}$ and the density of $k$-points sampling the Brillouin zone, were converged by means of a series of test calculations on bulk $\mathrm{Al}_{9} \mathrm{Co}_{2}$ (22 at/cell). The chosen values for $E_{\text {cut }}(350 \mathrm{eV})$ and the size of the Monkhorst-Pack $k$-points mesh $(8 \times 8 \times 8)$ achieve a precision for the bulk total energy smaller than $4 \mathrm{meV}$. For the calculations using slabs, we keep an identical k-grid mesh $(8 \times 8 \times 1)$.

The optimization of the bulk structure of the intermetallic compound $\mathrm{Al}_{9} \mathrm{Co}_{2}$ and the atomic structure model of the (001) surface has been fully described in reference [25]. Adsorption of atomic oxygen is simulated by building non-symmetric slabs separated by a $20 \AA$-thick vacuum region, where the position of the oxygen atom is relaxed starting from a $3 \AA$ distance above the surface site. Based on the results of Baker et al. [36, 37], it has been considered that the $20 \AA$-thick vacuum region is sufficient to ensure that the dipolar effect induced by the adsorbed oxygen atom on one side of the slab will not produce any important calculation artefact. Eleven-layer-thick slabs are used to perform the present study: the 5 atomic layers at the bottom are kept fixed while the other 6 top layers are allowed to fully relax (surface of interest). As shown in our previous study [25], this configuration is sufficient to obtain both accurate electronic and atomic 
structures. A comparison between the values of the relative adsorption energies obtained for different adsorption sites (which will be discussed in section 3.1) within an 11-layerthick slab and a 6-layer-thick slab shows contrasted differences: it is smaller than 40 meV for the bridge type sites $B_{1}, B_{2}$ and $B_{3}$, but of the order of $200 \mathrm{meV}$ for the sites $T_{1}$ and $H_{2}$ (see figure 1a). In the paper, we use a $1 \mathrm{x} 1$ surface unit cell. A comparison using a $2 \times 2$ surface unit cell indicates that the adsorption energy differences are small (smaller than $20 \mathrm{meV}$ ) for the considered adsorption sites $\left(B_{1}, B_{2}, B_{3}\right.$ and $H_{2}$ ). Our calculations were performed without taking into account spin-polarization effects on the obtained total energies. We have nevertheless verified that for different adsorption sites (namely $B_{2}$ and $B_{3}$, using a 6 layer thick slab with $2 \times 2$ surface unit cell), the total energy differences between calculations with and without spin-polarization are smaller than $1 \mathrm{meV}$.

\subsection{Experimental details}

The preparation of the $\mathrm{Al}_{9} \mathrm{Co}_{2}(001)$ surface consists of repeated cycles of $\mathrm{Ar}^{+}$ion sputtering for 30 minutes followed by annealing at different temperatures ranging from 843 to $1043 \mathrm{~K}$, all of this under ultra high vacuum conditions (base pressure $5 \times 10^{-11}$ mbar, see Ref. [25] for more details). The temperature has been measured using an infrared optical pyrometer with the emissivity set to 0.35 . To check the cleanliness and the overall structure of the surface, x-ray photoelectron spectroscopy (XPS) and low electron energy diffraction (LEED) have been used. The local atomic structure was investigated by STM (tungsten tip) and the electronic structure of the surface has been measured at room temperature using ultraviolet photoelectron spectroscopy (UPS) and scanning tunnelling spectroscopy (STS).

\section{Results and discussion}

\subsection{Identification of the most favoured sites for atomic oxygen adsorption}

The adsorption sites considered in this study are labelled Top $(T)$, Hollow $(H)$ and Bridge $(B)$, with 1-fold, 4-fold and 2-fold symmetry respectively. In the case of a $T_{i}$ site (where index $i=1$ to 2 labels all $T$ sites considered in this study), the adsorbed atom is lying over one $\mathrm{Al}$ atom in the substrate. In the case of a $B_{j}$ site (where index $j=1$ to 3 labels all $B$ sites considered in this study), the adsorbed atom forms a bridge between two $\mathrm{Al}$ atoms in the substrate, while in the case of a $H_{k}$ site (where index $k=1$ to 2 labels all $H$ sites considered in this study) the adsorbed atom is located in between four Al substrate atoms (see Fig. 1). The chosen sites are exhaustive for the studied surface termination and they present different local surface stoichiometries, i.e. with differences in the atomic chemical environment around the adsorption site. The aim of the present selection of the adsorption sites is to determine which are the parameters acting on the first steps of the oxidation of the $\mathrm{Al}_{9} \mathrm{Co}_{2}(001)$ surface. The relative adsorption energies 
$\Delta E^{(i)}$, for different adsorption sites $i$, are evaluated according to the equation:

$$
\Delta E^{(i)}=E_{\text {Slab }+O}^{(i)}-E_{\text {Slab+O }}^{B_{3}}
$$

where $E_{S l a b+O}^{(i)}$ is the total energy of the substrate-adsorbate system (slab + adsorbed oxygen atom in the site $i$ ) and $E_{S l a b+O}^{B_{3}}$ the total energy of the slab plus the oxygen atom adsorbed in the $B_{3}$ site.

Figure 1(a) presents the different adsorption sites considered in this study as well as the relaxed final positions of the oxygen atoms. Their corresponding relative adsorption energies are presented in Table 1. The most favourable adsorption site corresponds to the $B_{3}$ site (with a total adsorption energy of $-7.20 \mathrm{eV}$ with respect to the free oxygen atom, consitent with the atomic oxygen adsorption energy on $\mathrm{Al}(111)[38,39,15])$ followed by the $H_{1}^{\prime}$ site. Both sites are bridge sites. The site $B_{3}$ is also located between the cluster structures of the (001) surface termination (see Figure 1(b)). The adsorption energies of the $B_{2}, B_{1}$ and $T_{1}^{\prime}$ sites are calculated to be about $0.4 \mathrm{eV}$ higher than the one of the $B_{3}$ site. The $H_{2}, H_{2}^{\prime}$ and $T_{1}$ sites are found the less favourable sites. The oxygen atoms initially positioned in $H_{1}$ (equivalent to $H_{3}$ ), $T_{1}$ and $T_{2}$ (equivalent to $T_{3}$ ) relax towards $B_{3}$, whereas the atoms initially located on the sites $B_{1}, B_{2}$ and $B_{3}$ present only small relaxations. An oxygen atom initially deposited in $H_{2}$ relaxes out of the centre of the site (site $\left.H_{2}^{\prime}\right)$.

The oxygen atoms adsorbed on the surface show a tendency to relax towards adsorption sites leading to Al-O distances close to the one determined for oxygen adsorbed on $\mathrm{Al}(111)$ (1.73 - $1.84 \AA$ [40]) and similar to Al-O distances observed in $\mathrm{Al}_{2} \mathrm{O}(1.70 \AA[40])$ and $\mathrm{Al}_{2} \mathrm{O}_{3}(1.86 \AA[41])$ oxides. The Al-O distance calculated for oxygen atoms located in the $B_{3}$ site is $1.75 \AA$. Oxygen atoms adsorbed on the $T_{1}^{\prime}, H_{2}^{\prime}$ and $H_{1}^{\prime}$ sites cause important relaxations of the surface aluminium atoms (Figures 1(ce)): surface aluminium atoms rearrange around the adsorbed oxygen atom so that the latter, which was initially deposited in a 4-fold symmetry site, is finally located in a 3 -fold symmetry site formed by $3 \mathrm{Al}$ surface atoms. In the less favourable site $\left(H_{2}\right)$, the Al-O distance is $1.92 \AA$.

The adsorption energies depend strongly on the Co-O distance. The most favourable adsorption site $\left(B_{3}\right)$ is also the one that presents the largest Co-O separation $(3.95 \AA)$. In the less favourable site $\left(H_{2}\right)$, the oxygen atom is relatively close to a cobalt atom $(2.73 \AA)$. The relative adsorption energy of the $H_{2}^{\prime}$ site is unfavourable, the oxygen atom is positioned almost over a sub-surface cobalt atom. In general, we can conclude that a large $\mathrm{Co}-\mathrm{O}$ distance correlates with a more favourable adsorption site.

\subsection{Electronic structure of the adsorbate-substrate system: site $B_{3}$}

Figure 2 shows the local electronic density of states (LDOS) calculated for the surface before and after atomic oxygen adsorption. The effects of adsorption on the surface DOS are confined to the first two atomic layers (see Figure 2(left)). In the surface atomic 


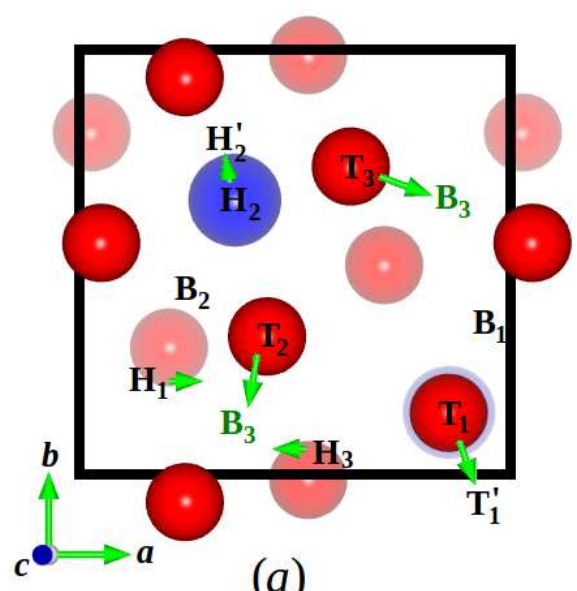

(a)

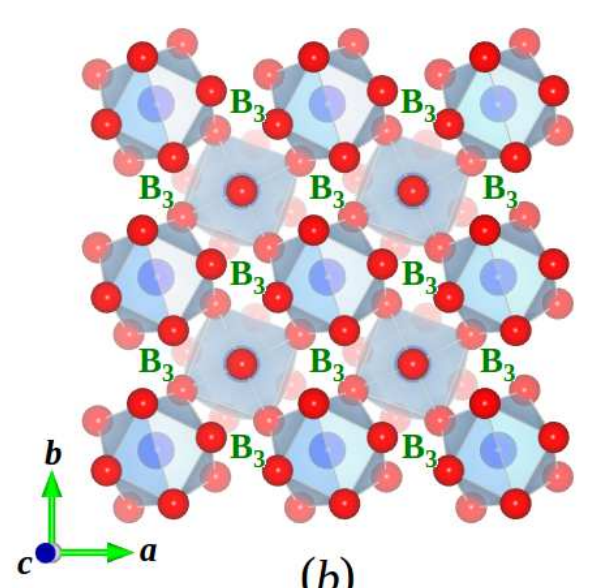

(b)

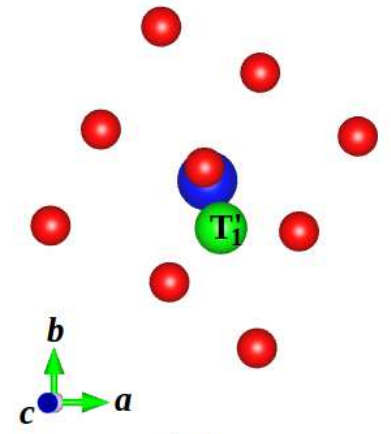

(c)

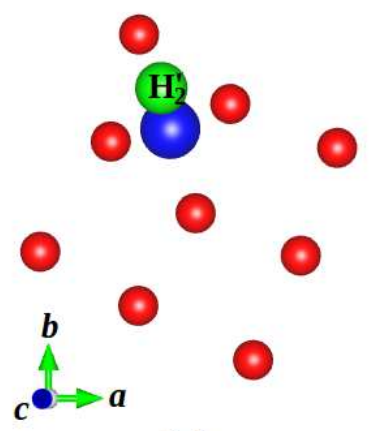

(d)

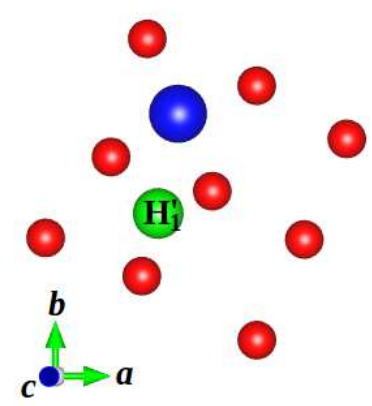

(e)

Figure 1. (a) Initial and final positions of the oxygen atoms adsorbed on the $\mathrm{Al}_{9} \mathrm{Co}_{2}$ (001) surface (top view, 1x1 surface unit cell represented in a black box). Red (blue) spheres represent the aluminium (cobalt) atoms. Sub-surface atoms appear hazed. The most important relaxations are indicated by green arrows and the most favourable adsorption site, $B_{3}$, is highlighted in green. (b) Cluster representation of the atomic structure of the $\mathrm{Al}_{9} \mathrm{Co}_{2}(001)$ surface. Substrate relaxations after atomic oxygen adsorption on the $\mathrm{Al}_{9} \mathrm{Co}_{2}(001)$ surface forming sites with almost 3-fold symmetry are shown for sites $T_{1}^{\prime}$ (c), $H_{2}^{\prime}$ (d) and $H_{1}^{\prime}$ (e). The blue sphere indicates the position of the nearest Co atom to the adsorbed oxygen atom (green spheres).

layers S and S-1, the corresponding structure of the DOS after adsorption differs from the DOS of the clean surface. Figure 2(middle) shows the details of the local DOS of the topmost surface atomic layer $\mathrm{S}$, as well as the contributions of the surface aluminium atoms and the adsorbed oxygen atom. Figure 2(right) shows a certain number of common structures between the partial electronic DOS of the substrate surface and the adsorbed oxygen atom (between the Fermi energy and -6 eV). Particularly, common peaks are observed at energies of $-5.9 \mathrm{eV}$ and $-3.8 \mathrm{eV},-2.7 \mathrm{eV},-1.0 \mathrm{eV}$ and $-0.4 \mathrm{eV}$. This strong mixing of the two sets of electronic states is characteristic of a covalent interaction, resulting in the hybridization between the electronic s-p states of the surface and the electronic s-p states of the oxygen atom. Furthermore, in the LDOS of the clean surface of Figure 2(right), a peak at $-2.0 \mathrm{eV}$ is observed. This particular peak disappears after the adsorption of the oxygen atom. In the clean surface, this structure is due to the 
Table 1. Relative adsorption energies $\Delta E^{(i)}$ of the different adsorption sites considered in the study.

\begin{tabular}{ccc} 
Initial Site & Final Site & $\Delta E^{(i)}[\mathrm{eV}]$ \\
\hline$B_{1}$ & $B_{1}$ & 0.40 \\
$B_{2}$ & $B_{2}$ & 0.34 \\
$B_{3}$ & $B_{3}$ & 0.00 \\
$H_{1}$ & $H_{1}^{\prime}\left(\right.$ near $\left.B_{3}\right)$ & 0.17 \\
$H_{2}$ & $H_{2}^{\prime}$ & 0.73 \\
$H_{2}$ & $H_{2}$ & 0.89 \\
$T_{1}$ & $T_{1}^{\prime}$ & 0.45 \\
$T_{1}$ & $T_{1}$ & 0.90 \\
$T_{2}$ & $B_{3}$ & 0.00 \\
\hline
\end{tabular}

hybridization between s-p states of the surface aluminium atoms and d states of the underlying sub-surface cobalt atom. It is possible to assume that the disappearance of this structure after the oxygen adsorption is due to the formation of an Al-O bond.

Figure 3 shows the charge density distribution of the adsorbed oxygen atom and one of the two aluminium atoms composing the $B_{3}$ site (the situation is the same for both $\mathrm{Al}$ atoms composing the $B_{3}$ site). In agreement with the previous findings, a nonuniform shape of the charge density is observed after oxygen adsorption. The deviation from spherical distribution of the charge density embedding the atoms point towards the formation of a covalent and directional bonding between the surface $\mathrm{Al}$ atoms and the adsorbed $\mathrm{O}$ atom, since the flattening of the charge density occurs in between these atoms.

\subsection{Comparison between calculations and experimental observations by STM}

Figure 4(a) shows an experimental STM image recorded at $300 \mathrm{~K}$ after adsorption of molecular oxygen on the $\mathrm{Al}_{9} \mathrm{Co}_{2}(001)$ surface. Particularly, this STM image shows the substrate lattice as well as adsorbates with three different types of contrasts: (a) dark squares with a bright centre, (b) dark zones, and (c) bright dots. The number of motifs of type "b" and "c" increases with $\mathrm{O}_{2}$ exposure while the number of motifs "a" remains constant. These assessments are deduced after the analysis of the experimental STM images, by measuring the areas of the image covered by motifs of type "a", "b" and "c" as a function of the $\mathrm{O}_{2}$ dosage (Fig. 4(b)). Motifs of type "a" can already be observed on the bare substrate before $\mathrm{O}_{2}$ exposure, and do not present any mobility. Therefore, we assume that type "a" corresponds to impurities or vacancies already present on the sample surface. Analysing consecutive STM scans recorded over the same region, it has been observed that several "c" motifs apparently disappear to be replaced by "b" motifs at the same site. At this stage, it is not clear if these "c" motifs diffuse across terraces between scans or transform into "b" motifs. For a limited number of cases, "c" motifs 

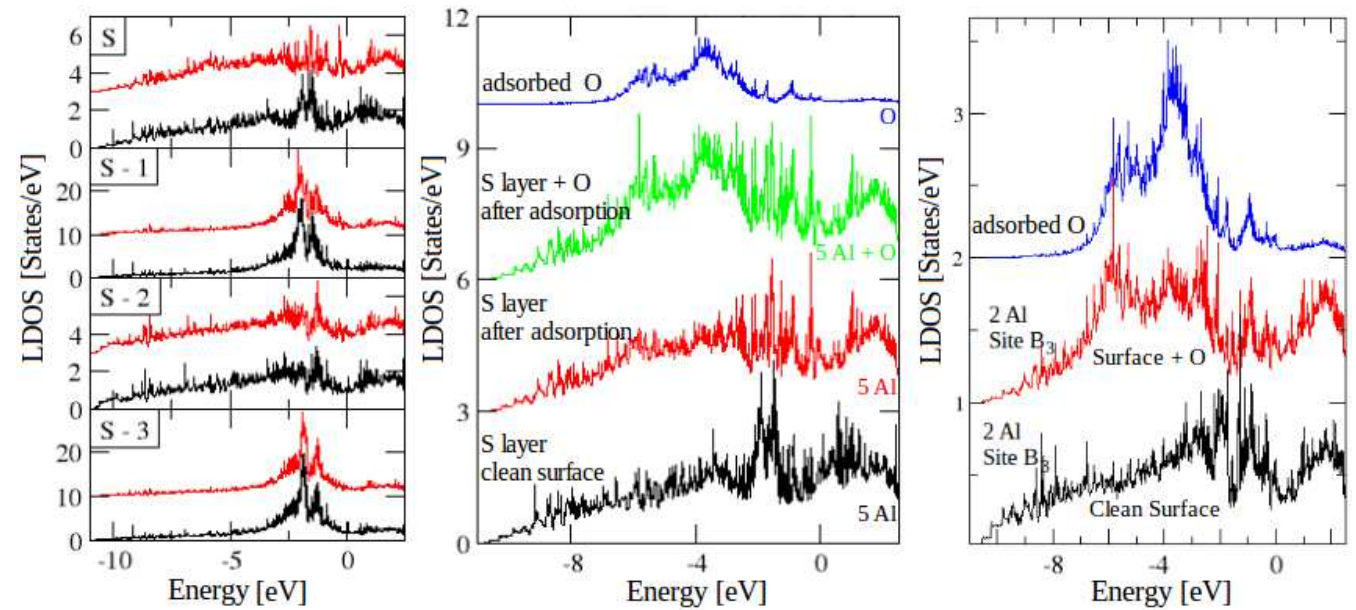

Figure 2. Left: Electronic LDOS structure of the atomic layers of the clean surface (black) and of the slab after atomic oxygen adsorption (red, site $B_{3}$ ); Middle: Different contributions to the electronic structure of the surface plane $S$ (5 aluminium atoms per surface unit cell) and the adsorbed oxygen atom ( $B_{3}$ site). Right: Partial LDOS (i) of the two $\mathrm{Al}$ atoms composing the $B_{3}$ site (black, clean surface), (ii) of the two $\mathrm{Al}$ atoms of the $B_{3}$ site after oxygen adsorption (red), (iii) of the adsorbed oxygen atom in the $B_{3}$ site (blue).
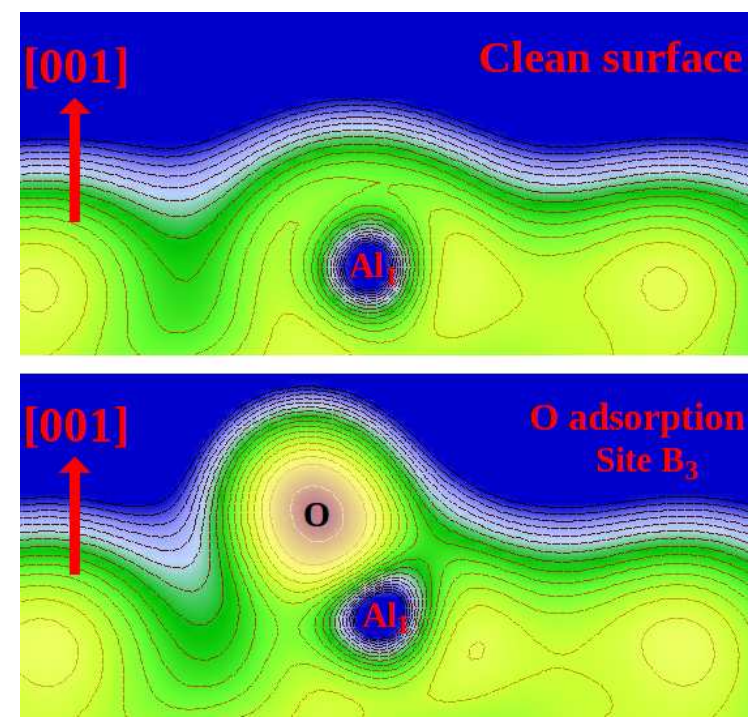

Figure 3. Slice cuts of the charge density distribution of the site $B_{3}$, composed of two $\mathrm{Al}$ atoms (only one shown), before (clean surface) and after atomic oxygen adsorption.

have appeared on top of already existing "b" motifs. These observations are consistent with the dissociative adsorption of $\mathrm{O}_{2}$ molecules at the surface, the "b" and "c" motifs corresponding to oxygen atoms and/or $\mathrm{O}_{2}$ molecules adsorbed on the surface.

Figure 5 presents the STM images that were simulated using the Tersoff-Hamann approximation $[19,20]$ for the adsorption of atomic oxygen in the $H_{2}, H_{1}^{\prime}, B_{3}$ and $T_{1}$ sites. The simulated images corresponding to the adsorption of oxygen in $H_{2}$ and $T_{1}$ 


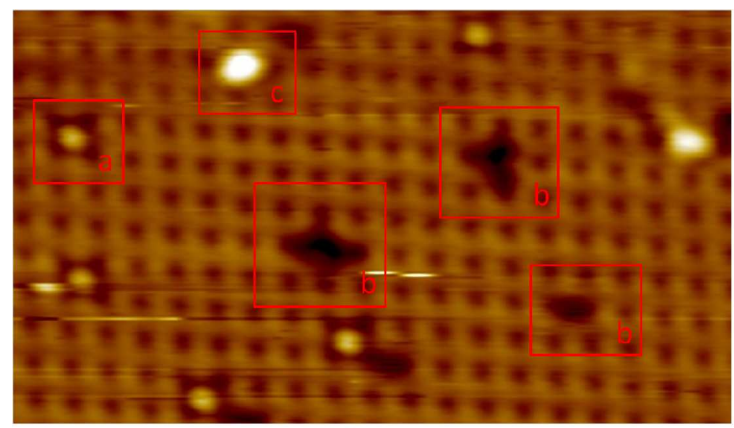

(a)

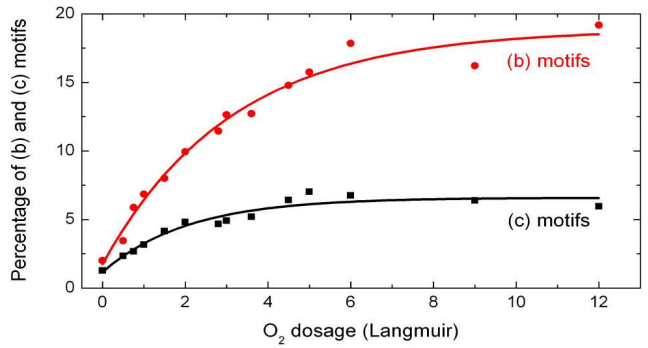

(b)

Figure 4. (a) Experimental STM image recorded at $300 \mathrm{~K}$ for an exposure of 0.5 Langmuir of $\mathrm{O}_{2}$ deposition $\left(9.6 \times 12.1 \mathrm{~nm}^{2}, V_{\text {bias }}=-2 \mathrm{~V}\right)$. Red boxes identify the different motifs "a", "b" and "c" observed in the STM image. (b): Percentage of "b" and "c" motifs observed after $\mathrm{O}_{2}$ dosage (in Langmuir).

sites show a bright point, that does not necessarily correspond to the position of the adsorbed atomic oxygen (red arrow on Fig. 5). From an energetic point of view, the sites $H_{2}$ and $T_{1}$ are not favourable, and the relative position of the bright spot with respect to the underlying pattern of the bare surface is different for the experimental and the simulated STM images. The motifs of type "c" might rather correspond to molecular oxygen adsorption, but precise determination of this point requires further studies. The simulated STM images corresponding to the adsorption of oxygen in site $B_{3}$ or $H_{1}^{\prime}$ shows large dark areas, similarly to motif "b" of the experimental image. In addition, these two sites are the most favourable from an energetic point of view. This might allow us to interpret the motif "b" on the experimental STM image as the presence of atomic oxygen adsorbed in site $B_{3}$ or $H_{1}^{\prime}$.

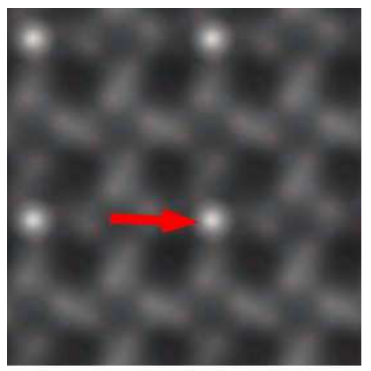

$\mathrm{H}_{2}$

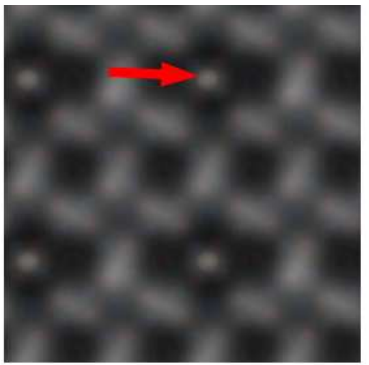

$\mathrm{B}_{3}$

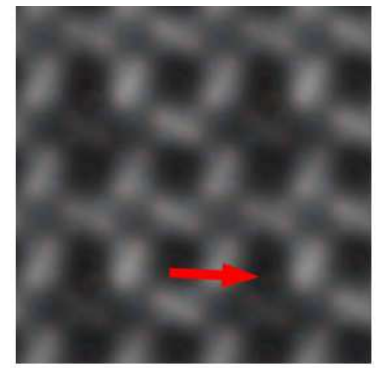

$\mathrm{H}_{1}$

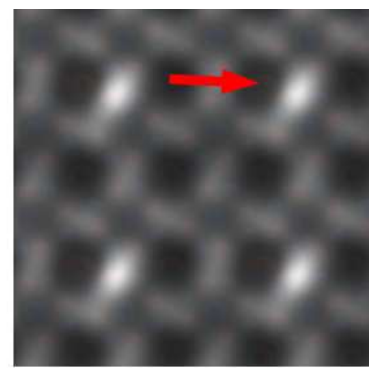

$\mathrm{T}_{1}$

Figure 5. Simulated STM images of the atomic oxygen adsorption on the $H_{2}, B_{3}$, $H_{1}^{\prime}$ and $T_{1}$ sites. The tip-surface distance is $4 \AA$ and $V_{\text {bias }}=-1.3 \mathrm{~V}(2 \times 2$ surface unit cell). The red arrow show the position of the adsorbed oxygen atom. 


\section{Conclusions}

In this article, the atomic oxygen adsorption on the (001) surface of the $\mathrm{Al}_{9} \mathrm{Co}_{2}$ intermetallic compound has been studied by means of calculations based on the DFT framework and experimental STM images. The preferential adsorption site has been identified as a bridge-type site between the cluster structures of the (001) surface termination ( site $B_{3}$ ) and equidistant from the sub-surface Co atoms. The Al-O bonding between adsorbate and substrate shows a covalent behaviour, with hybridization between the oxygen s-p states and the aluminium s-p states of the surface. The simulated STM image from a slab containing one adsorbed oxygen atom in the preferential adsorption site presents a dark contrast in agreement with what is observed in the experimental STM images. In this study, it has also been shown that the oxygen adsorption generates important atomic relaxations of the topmost layer of the surface: oxygen atoms cause a rearrangement of the initial 4-fold sites, turning them into 3fold sites. It has also been found that only weak relaxations are obtained when the oxygen atom is adsorbed on a bridge site: the adsorbed oxygen atoms suffer almost no relaxation in the directions parallel to the surface.

Finally, this work has shown that sub-surface cobalt atoms are found to strongly influence the values of the adsorption energies: the most favoured sites lie at the longest Co-O distances $(\simeq 3.95 \AA)$. Al-O distances are rather short in comparison with Co-O distances. When adsorbed in $B_{3}$ site, the Al-O distance is the smallest one $(\simeq 1.75$ $\AA)$. The obtained Al-O distances are in agreement with the typical distances of oxygen adsorption on the $\mathrm{Al}(111)$ surface, as well as with the $\mathrm{Al}-\mathrm{O}$ distances in the $\mathrm{Al}_{2} \mathrm{O}$ and $\mathrm{Al}_{2} \mathrm{O}_{3}$ oxides. Therefore, the formation of these oxides is expected on the (001) surface of the $\mathrm{Al}_{9} \mathrm{Co}_{2}$ compound. From our experimental results, these oxides would be disordered since the LEED pattern disappears when a rate of 17 langmuirs of $\mathrm{O}_{2}$ is deposited on the surface at $300 \mathrm{~K}$.

\section{Acknowledgements}

The "Agence Nationale de la Recherche" (ANR-08-BLAN-0041-01 and ANR-11-INTB1001-01) is acknowledged for its financial support. This work was granted access to the HPC resources of the French institute IDRIS (Institute du Développement et des Ressources en Informatique Scientifique) under allocation 2010-99642 made by GENCI (Grand Equipement National de Calcul Intensif). S.A.V. thanks INC-CNRS and the "Région Lorraine" for financial support during his Ph.D. thesis.

\section{References}

[1] E. Belin-Ferré, editor. Surface properties and engineering of complex intermetallics, volume 3. World Scientific Publishing, Singapore, 2010.

[2] K. Reuter, C. Stampfl, M.V. Ganduglia-Pirovano, and M. Scheffler. Atomistic description of oxide formation on metal surfaces: the example of ruthenium. Chem. Phys. Letters, 352:311, 2002. 
[3] R.W. Joyner and R.A. Van Santen, editors. Elementary Reaction Steps in Heterogeneous Catalysis. NATO ASI. Series C, Kluwer Academic Pub., Dordrecht, 1993.

[4] G. Katz, R. Kosloff, and Y. Zeiri. Abstractive dissociation of oxygen over Al(111): A nonadiabatic quantum model. J. Chem. Phys., 8:3931, 2004.

[5] L. Osterlund, I. Zoric, and B. Kasemo. Dissociative sticking of $\mathrm{O}_{2}$ on $\mathrm{Al}(111)$. Phys. Rev. B, 55:15452, 1997.

[6] V. Rose, V. Podgursky, I. Costina, and R. Franchy. Growth of ultra-thin amorphous $\mathrm{Al}_{2} \mathrm{O}_{3}$ films on $\operatorname{CoAl}(100)$. Surf. Sci., 541:128, 2003.

[7] V. Rose, V. Podgursky, I. Costina, R. Franchy, and H. Ibach. High temperature oxidation of CoAl(100). Surf. Sci., 577:139, 2005.

[8] R. Franchy. Growth of thin, crystalline oxide, nitride and oxynitride films on metal and metal alloy surfaces. Surf. Sci. Report, 38:199, 2000.

[9] G. Schmitz, M. Eumann, D. Stapel, and R. Franchy. Combined EELS STM study of the adsorption of nitric oxide and the formation of $\mathrm{GaO}_{x} \mathrm{~N}_{y}$ on CoGa(001). Surf. Sci., 91:427, 1999.

[10] M. Schmid, G. Leonardelli, R. Tscheliessnig, A. Biedermann, and P. Varga. Oxygen adsorption on $\operatorname{Al}(111)$ : low transient mobility. Surf. Sci., 478:355, 2001.

[11] C. Carbogno, J. Behler, K. Reuter, and A. Gross. Signatures of nonadiabatic $\mathrm{O}_{2}$ dissociation at $\operatorname{Al}(111)$ : First-principles fewest-switches study. Phys. Rev. B, 81:035410, 2010.

[12] H. Brune, J. Wintterlin, R.J. Behm, and G. Ertl. Surface migration of hot adatoms in the course of dissociative chemisorption of oxygen on Al(111). Phys. Rev. Lett., 68:624, 1992.

[13] H. Brune, J. Wintterlin, J. Trost, G. Ertl, J. Wiechers, and R.J. Behm. Interaction of oxygen with $\mathrm{Al}(111)$ studied by scanning tunnelling microscopy. J. Chem. Phys., 99:2128, 1993.

[14] G. Wahnstrom, A.B. Lee, and J. Stromquist. Motion of "hot" oxygen adatoms on corrugated metal surfaces. J. Chem. Phys., 105:326, 1996.

[15] Y. Yourdshahyan, B. Razaznejad, and B.I. Lundqvist. Adiabatic potential-energy surfaces for oxygen on $\mathrm{Al}(111)$. Phys. Rev. B, 65:075416, 2002.

[16] R. Wiesendanger and H.-J. Güntherodt, editors. Scanning Tunneling Microscopy III. Springer series in Surface Science 29, Springer Verlag, Berlin, 1993.

[17] W.A. Hofer. Challenges and errors: interpreting high resolution images in scanning tunneling microscopy. Prog. Surf. Sci., 71:147, 2003.

[18] J.M. Blanco, F. Flores, and R. Prez. STM-theory: Image potential, chemistry and surface relaxation. Prog. Surf. Sci., 81:403, 2006.

[19] J. Tersoff and D. R. Hamann. Theory and application for the scanning tunneling microscope. Phys. Rev. Lett., 50:1998, 1983.

[20] J. Tersoff and D. R. Hamann. Theory of the scanning tunnelling microscope. Phys. Rev. B, 31:805, 1985.

[21] E. Belin-Ferré, editor. Basics of Thermodynamics And Phase Transitions In Complex Intermetallics, volume 1. World Scientific Publishing, Singapore, 2008.

[22] E. Belin-Ferré, editor. Properties and Applications of Complex Intermetallics, volume 2. World Scientific Publishing, Singapore, 2009.

[23] E. Belin-Ferré, editor. Mechanical Properties of Complex Intermetallics, volume 4. World Scientific Publishing, Singapore, 2010.

[24] K. H. J. Buschow, R. W. Cahn, M. C. Flemings, B. Ilschner, E. J. Kramer, Subhash Mahajan, and P. Veyssire, editors. Encyclopedia of Materials: Science and Technology. Elsevier Science Ltd., NY, 2001.

[25] S. Alarcón Villaseca, J. Ledieu, L.N. Serkovic Loli, M.-C. de Weerd, P. Gille, V. Fournée, J.-M. Dubois, and É. Gaudry. Structural investigation of the (001) surface of the $\mathrm{Al}_{9} \mathrm{Co}_{2}$ complex metallic alloy. J. Phys. Chem. C, 115:14922, 2011.

[26] D. Plachke, A. Khellaf, and H.D. Carstanjen. Initial oxidation of AlPdMn quasicrystals a study by high-resolution RBS and ERDA. Nuclear Instruments and Methods in Physics Research B, 190:646, 2002. 
[27] D. Plachke, A. Khellaf, M. Kurth, A. Szokefalvi-Nagy, and H.D. Carstanjen. Initial oxidation of quasicrystals an investigation by high-resolution RBS and ERDA. Journal of Non-Crystalline Solids, 334-335:524, 2004.

[28] A. Lekatou, A.K. Skas, A.E. Karantzalis, and D. Sioulas. Microstructure and corrosion performance of Al-32\%Co alloys. Corrosion Science, 63:193, 2012.

[29] A.I. Goldman, D. Sordelet, P. A. Thiel, and J. M. Dubois, editors. New Horizons in Quasicrystals: Research and Applications. World Scientific Publishing Co, Singapore, 1997.

[30] G. Kresse and J. Furthmüller. Efficient iterative schemes for ab initio total-energy calculations using a plane-wave basis set. Phys. Rev. B, 54:11169, 1996.

[31] G. Kresse and J. Furthmüller. Efficiency of ab-initio total energy calculations for metals and semiconductors using a plane wave basis set. Comp. Mater. Sci., 6:15, 1996.

[32] P.E. Blöchl. Projector augmented-wave method. Phys. Rev. B, 50:17953, 1994.

[33] G. Kresse and D. Joubert. From ultrasoft pseudopotentials to the projector augmented-wave method. Phys. Rev. B, 59:1758, 1999.

[34] J. P. Perdew, K. Burke, and M. Ernzerhof. Generalized gradient approximation made simple. Phys. Rev. Lett., 77:3865, 1996.

[35] J. P. Perdew, K. Burke, and M. Ernzerhof. Erratum: Generalized gradient approximation made simple. Phys. Rev. Lett., 78:1396, 1997.

[36] T.A. Baker, C.M. Friend, and E. Kaxiras. Chlorine interaction with defects on the Au (111) surface: a first-principles theoretical investigation. J. Chem. Phys., 129:104702, 2008.

[37] T.A. Baker, C.M. Friend, and E. Kaxiras. Effects of chlorine and oxygen coverage on the structure of the Au (111) surface. J. Chem. Phys., 130:084701, 2009.

[38] A. Kiejna and B. I. Lundqvist. First-principles study of surface and subsurface o structures at al(111). Phys. Rev. B, 63:085405, Feb 2001.

[39] A. Kiejna and B.I. Lundqvist. Stability of oxygen adsorption sites and ultrathin aluminum oxide films on al(111). Surface Science, 504:1-10, 2002.

[40] A. Benali. Étude ab initio d'alliages AlCu: phénomènes de ségrégation et modification de la réactivité de surface vis-à-vis de l'oxygène. PhD thesis, École doctorale Science de la Matière, Toulouse, 2010.

[41] W.B. Pearson, I.D. Brown, and A.Mc.L. Mathieson. Structure Reports, 27, 1962. 